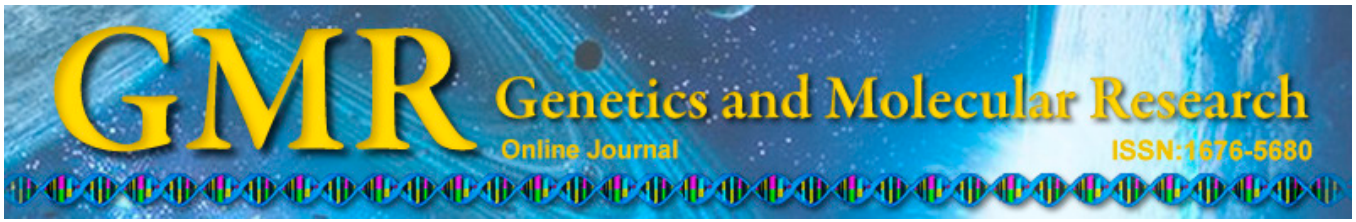

\title{
IL-17A and IL-17F polymorphisms and gastric cancer risk: a meta-analysis
}

\author{
Z. Li, Y. Liu, D. Cao, M. Jiang and F. Luo \\ Lung Cancer Center, Cancer Center, State Key Laboratory of Biotherapy, \\ West China Hospital of Sichuan University, Chengdu, China \\ Corresponding author: F. Luo \\ E-mail: luofeng_scu@yeah.net \\ Genet. Mol. Res. 14 (2): $7008-7017$ (2015) \\ Received September 3, 2014 \\ Accepted January 21, 2015 \\ Published June 26, 2015 \\ DOI http://dx.doi.org/10.4238/2015.June.26.10
}

\begin{abstract}
We conducted a meta-analysis of eligible studies to estimate the association between gastric cancer risk and rs2275913G $>$ A IL-17A and rs763780T $>$ C IL-17F polymorphisms. We searched the relevant studies in both Chinese and English through PubMed, the Web of Science, the Cochrane Library, and EMBASE up to January 1, 2014, including 3939 cases and 5407 controls. Seven eligible case-control studies were selected, including seven studies on rs $2275913 \mathrm{G}>\mathrm{A}$ IL-17A and four studies on rs763780T $>$ C IL-17F. The rs 2275913 AG [odds ratio $(\mathrm{OR})=1.50,95 \%$ confidence interval $(95 \% \mathrm{CI})=1.04-2.15]$ and $\mathrm{GG}(\mathrm{OR}=1.40,95 \% \mathrm{CI}=$ 1.00-1.96) genotypes were significantly associated with increased risk of gastric cancer compared with the AA genotype. The rs763780 TC $(\mathrm{OR}=$ $1.47,95 \% \mathrm{CI}=1.32-1.64)$ and $\mathrm{TT}(\mathrm{OR}=1.49,95 \% \mathrm{CI}=1.11-1.99)$ genotypes can influence gastric cancer risk. Subgroup analysis showed that rs $2275913 \mathrm{GG}(\mathrm{OR}=1.35,95 \% \mathrm{CI}=1.05-1.73)$ and $\mathrm{rs} 763780 \mathrm{TC}(\mathrm{OR}=$ $1.44,95 \% \mathrm{CI}=1.20-1.75)$ genotypes were not significantly associated with increased risk of gastric cancer in Japanese populations. Our meta-analysis is the first to indicate that the $\mathrm{rs} 2275913 \mathrm{G}>\mathrm{A}$ and $\mathrm{rs} 763780 \mathrm{~T}>\mathrm{C}$ polymorphisms are risk factors for gastric cancer development.
\end{abstract}

Key words: IL-17A; IL-17F; Polymorphism; Gastric cancer 


\section{INTRODUCTION}

Gastric cancer is one of the most common malignant diseases, and the fifth most common malignancy worldwide. It is estimated that 952,000 new gastric cancer cases and 723,000 deaths occurred in 2012. More than 70\% of gastric cancer cases occur in developing countries, and half of these have occurred in China (IARC, 2014). The etiology of gastric cancer is not well understood. Chronic Helicobacter pylori infection is considered an important risk factor for gastric cancer, but H. pylori infection is not a sufficient risk factor (IARC, 1994), since only a few H. pylori-infected people develop gastric cancer during their lifetime ( $\mathrm{Lu}$ and $\mathrm{Li}$, 2014). Previous epidemical studies have shown that drinking alcohol, being overweight, and high intake of salt are the risk factors for developing gastric cancer. However, high intake of fruit and vegetables with some antioxidants, vitamins, minerals, and beta-carotene protects against gastric cancer (van den Brandt and Goldbohm, 2006).

It has been reported that chronic inflammation is a risk factor for malignant transformation, but the role inflammation plays in cancer initiation is still not completely understood (Scheller et al., 2006; Candido and Hagemann, 2013). The interleukin-17 (IL-17) family of cytokines comprises six protein members, i.e., IL-17A, IL-17B, IL-17C, IL-17D, IL-17E, and IL-17F. IL-17A and IL-17F are the most important members; they all located at $6 \mathrm{q} 12$ and comprise three exons and two introns (Iwakura et al., 2011). Previous studies have shown that over-expression of IL-17A and IL-17F contributes to the development of various cancers, such as pancreatic, gastric, and lung cancers (Cully, 2014; Dai et al., 2014; Zarogoulidis et al., 2014; Kaabachi et al., 2014).

Several previous studies have reported an association between rs2275913G $>$ A IL-17A and rs $763780 \mathrm{~T}>\mathrm{C}$ IL-17F and the risk of gastric cancer, but the results are inconsistent. Therefore, we conducted a meta-analysis of eligible studies to estimate the association between gastric cancer risk and $\mathrm{rs} 2275913 \mathrm{G}>\mathrm{A}$ and $\mathrm{rs} 763780 \mathrm{~T}>\mathrm{C}$ polymorphisms.

\section{MATERIAL AND METHODS}

\section{Search strategy}

A comprehensive electronic search was conducted on May 1st, 2014, through the databases: PubMed, EMBASE, and the China National Knowledge Infrastructure platforms. The electronic search was performed using the following search terms: "Interluekin-17," "IL-17," "polymorphism", "variant", "gastric cancer," and "stomach cancer." The related articles were retrospective to identify additional potential studies. The reference lists of articles included for review and past meta-analyses were examined for any further relevant publications. No publication date or language restrictions were applied.

\section{Study selection}

The inclusion criteria for studies were as follows: the studies were of case-control design; the studies evaluated the association between gastric cancer risk and IL-17 polymorphisms; and the studies reported sufficient genotype frequencies to estimate odds ratios (ORs) and $95 \%$ confidence intervals (CIs).

The exclusion criteria for studies were as follows: articles only having an abstract, 
review articles, and comments; studies overlapping with other studies; studies having no comparison or control group; and studies having no data on genotype frequencies.

Corresponding authors were contacted in an attempt to obtain unreported genotype counts if studies were otherwise eligible.

\section{Data extraction}

Information was carefully extracted from all collected studies independently by two reviewers according to the inclusion criteria. Duplications and obviously irrelevant studies were excluded according to the exclusion criteria. We extracted the full texts of all eligible studies according to the inclusion criteria. The first author's name, publication data, sources of controls, numbers of gastric cancer cases, and controls with different genotypes were extracted for each eligible study.

\section{Statistical analysis}

All meta-analyses were conducted by the STATA 9.0 software. The Hardy-Weinberg equilibrium of the control group was assessed. ORs with $95 \%$ CIs were calculated to evaluate the strength of the association between the rs2275913G $>$ A and rs $763780 \mathrm{~T}>\mathrm{C}$ polymorphisms and gastric cancer risk. For the rs $2275913 \mathrm{G}>\mathrm{A}$ polymorphism, the pooled ORs were obtained for AG vs AA and GG vs AA. For the rs763780T $>$ C polymorphism, the pooled ORs were obtained for CT vs TT and CC vs TT. Subgroup analysis with ethnicity was also analyzed statistically. The heterogeneity between studies was estimated by the $\mathrm{I}^{2}$ test and heterogeneity Q statistic test. When $\mathrm{I}^{2}$ values were in the range $0-25 \%$, there was no heterogeneity; when $\mathrm{I}^{2}$ values were in the range of $25-50 \%$, there was moderate heterogeneity; and when $\mathrm{I}^{2}$ values were in the range of $75-100 \%$, there was a large degree of heterogeneity. A random-effect or fixed-effect model was taken to calculate the pooled ORs $(95 \% \mathrm{CI}) \mathrm{ac}-$ cording to the degree of heterogeneity between studies. The publication bias in studies was calculated using a funnel plot.

\section{RESULTS}

Our comprehensive literature search identified a total of 33 studies for the association between rs2275913G $>$ A and rs763780 $>C$ and gastric cancer risk based on their titles. Eventually, seven eligible case-control studies were selected, including seven studies for rs $2275913 \mathrm{G}>\mathrm{A}$ and four studies for $\mathrm{rs} 763780 \mathrm{~T}>\mathrm{C}$. Among the 26 studies excluded, two were dissertations, three were reviews, seven did not research $\mathrm{rs} 2275913 \mathrm{G}>\mathrm{A}$ and $\mathrm{rs} 763780 \mathrm{~T}>\mathrm{C}$ polymorphisms, 12 did not report the association between $\mathrm{rs} 2275913 \mathrm{G}>\mathrm{A}$ and $\mathrm{rs} 763780 \mathrm{~T}>\mathrm{C}$ polymorphisms and gastric cancer risk, and two were duplicate studies.

The characteristics of the seven included studies are summarized in Table 1 (Shibata et al., 2009; Chen, 2010; Wu et al., 2010; Arisawa et al., 2012; Rafiei et al., 2013; Zhang et al., 2014; Qinghai et al., 2014) and Table 2 (Shibata et al., 2009; Wu et al., 2010; Zhang et al., 2014). 
Z. Li et al.

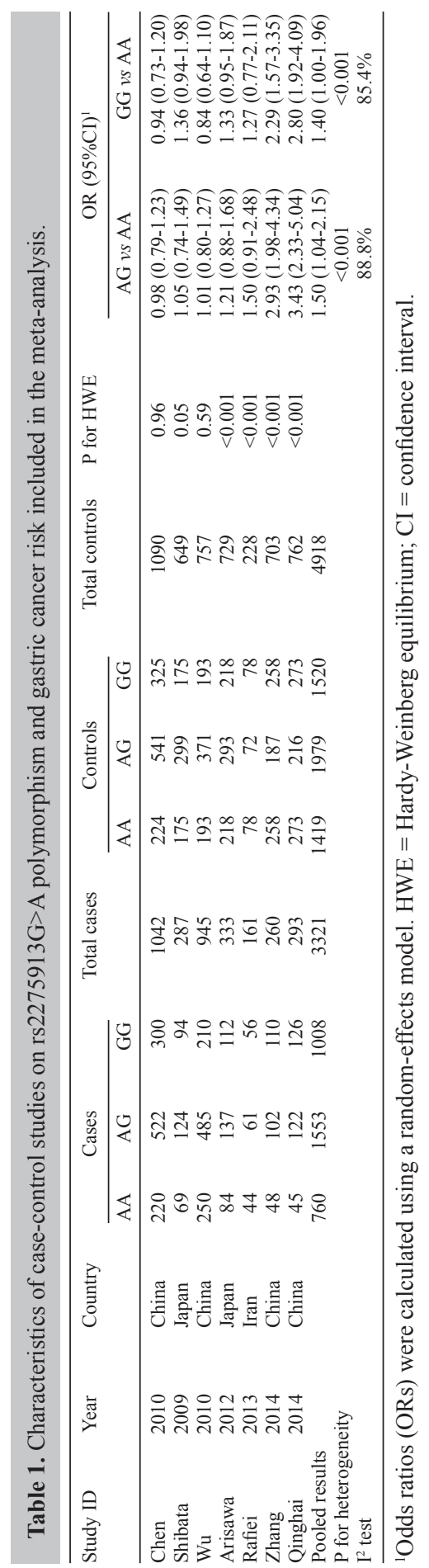

Genetics and Molecular Research 14 (2): $7008-7017$ (2015) 


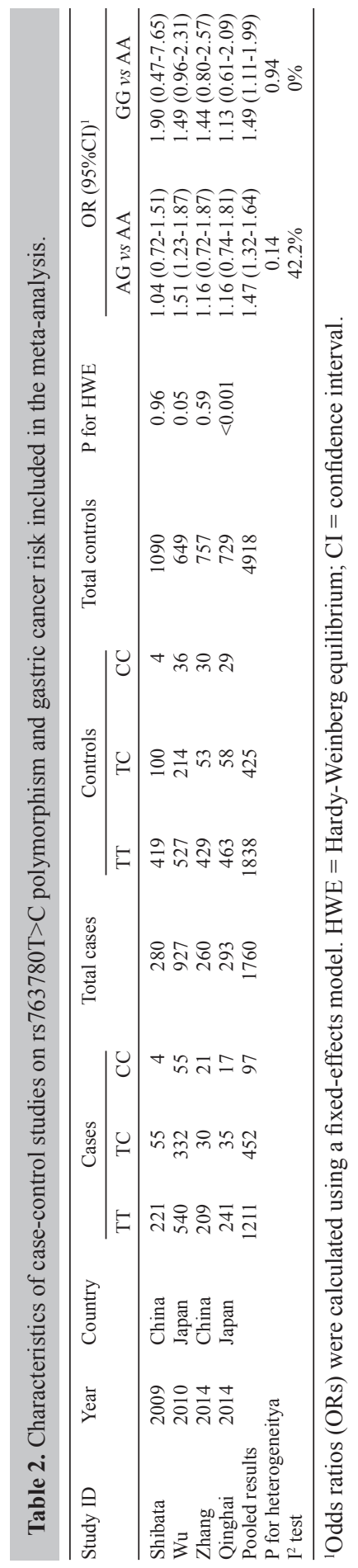


Seven studies reported the association between the rs $2275913 \mathrm{G}>$ A polymorphism and gastric cancer risk, including 3321 gastric cancer cases and 4918 controls. Eleven studies reported the association between the rs $763780 \mathrm{~T}>\mathrm{C}$ polymorphism and gastric cancer risk, including 3456 gastric cancer cases and 4957 controls. They were published between 2007 and 2013. Four studies were conducted in a Chinese population, two studies in a Japanese population, and one study in an Iranian population.

Four studies regarding rs2275913G $>$ A (Arisawa et al., 2012; Rafiei et al., 2013; Zhang et al., 2014; Qinghai et al., 2014) and one study regarding rs763780T $>C$ (Qinghai et al., 2014) did not accord with the Hardy-Weinberg equilibrium. There was a significant heterogeneity between pooled studies in terms of $\mathrm{rs} 2275913 \mathrm{G}>\mathrm{A}$, and thus we used a random-effect model to assess the association between rs $2275913 \mathrm{G}>\mathrm{A}$ and gastric cancer risk. Our meta-analysis found that rs $2275913 \mathrm{AG}$ and $\mathrm{GG}$ genotypes were significantly associated with increased risk of gastric cancer compared with the AA genotype, and the pooled ORs $(95 \% \mathrm{CI})$ were 1.50 (1.04-2.15) and 1.40 (1.00-1.96), respectively. Moreover, we found that rs763780 TC and TT genotypes can influence gastric cancer risk, and the ORs $(95 \% \mathrm{CI})$ were $1.47(1.32-1.64)$ and 1.49 (1.11-1.99), respectively.

Subgroup analysis showed that the rs2275913 GG genotype was not significantly associated with increased risk of gastric cancer in Japanese populations, with an OR $(95 \% \mathrm{CI})$ of 1.35 (1.05-1.73) (Figure 1). Similarly, we found that the rs763780 TC genotype was significantly associated with increased gastric cancer risk in the Japanese population, with an OR $(95 \% \mathrm{CI})$ of 1.44 (1.20-1.75) (Figure 2).

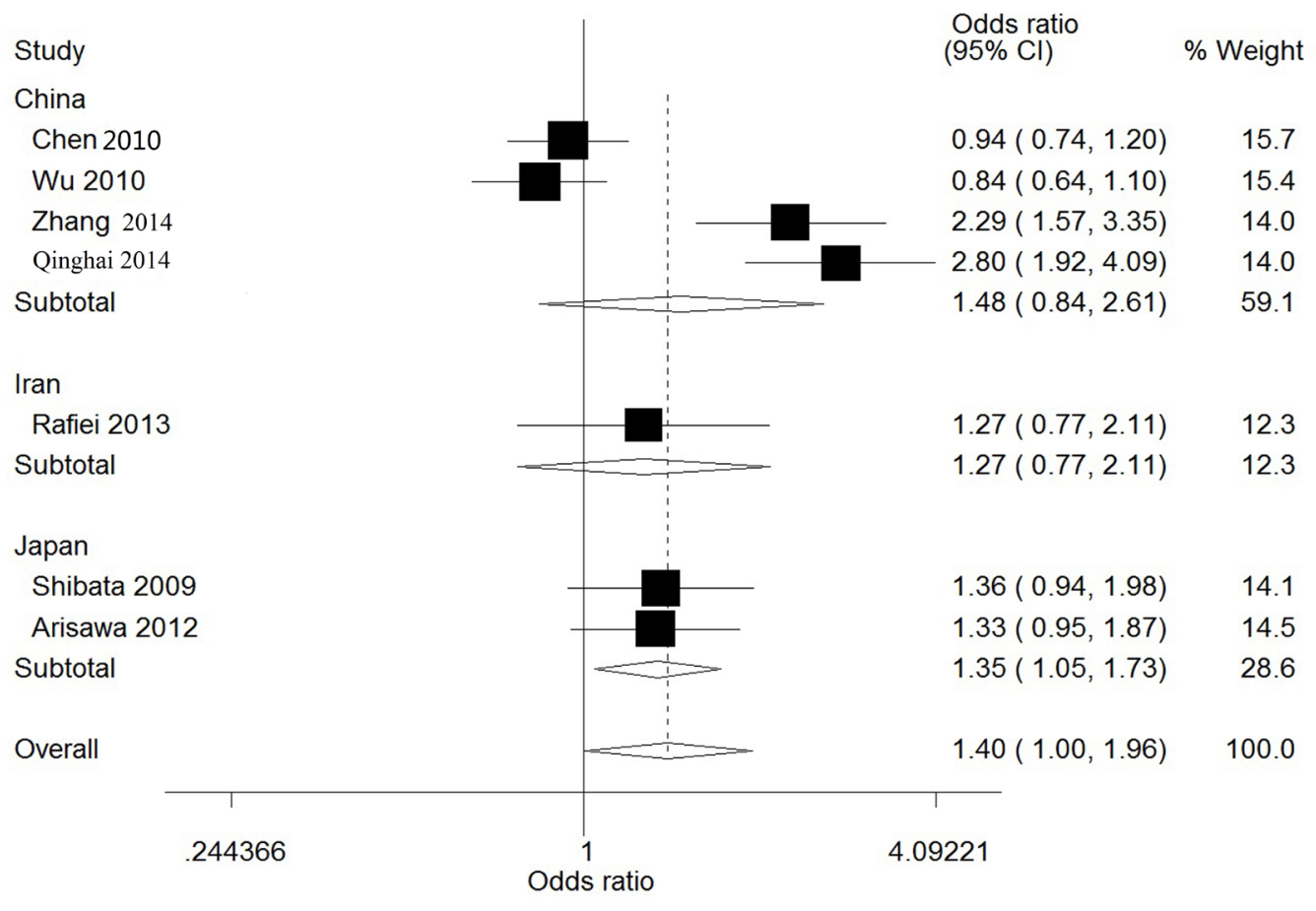

Figure 1. Subgroup analysis for association between rs2275913 GG and gastric cancer risk. 


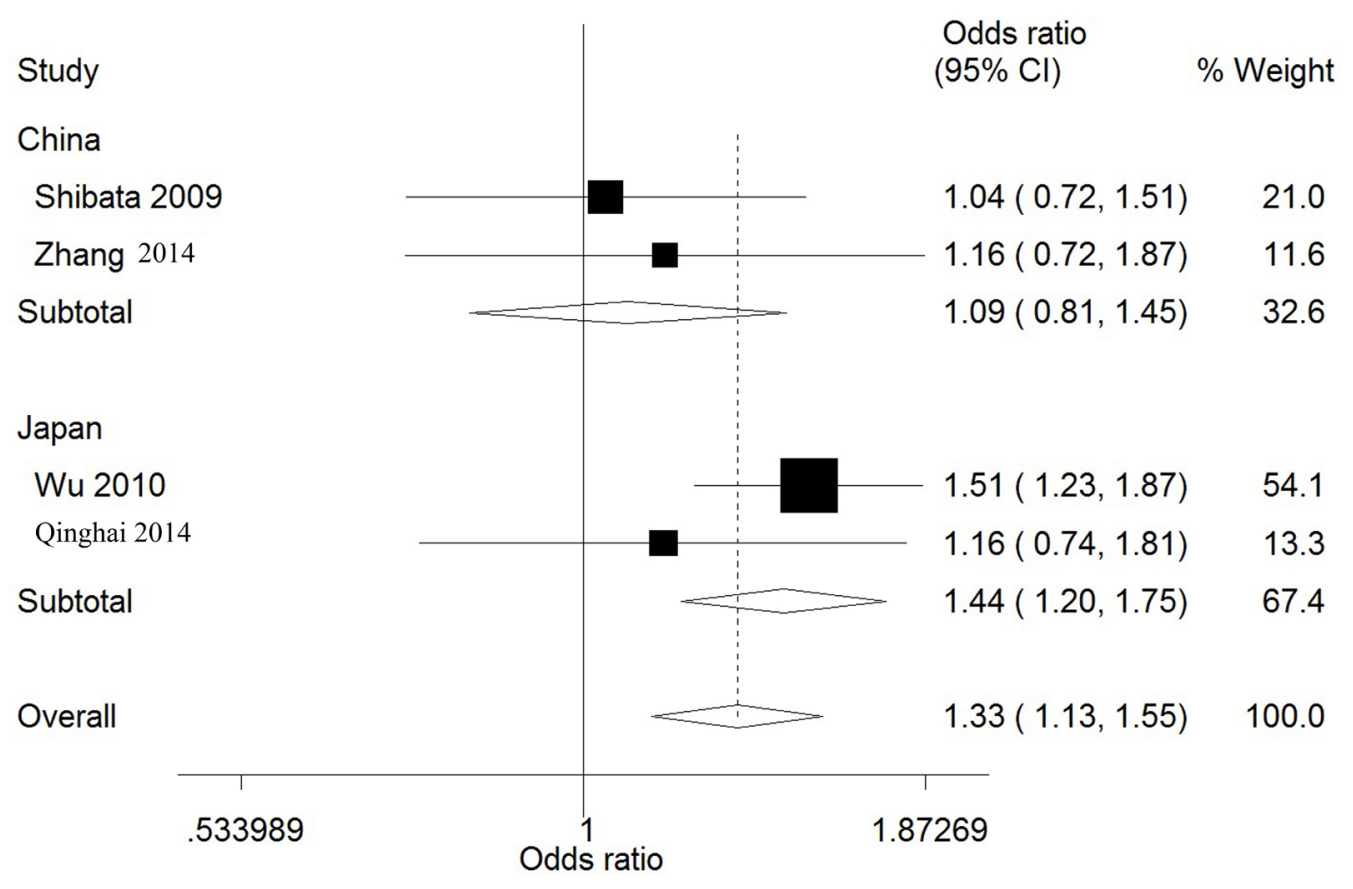

Figure 2. Subgroup analysis for association between rs 763780 TC and gastric cancer risk.

The shapes of the Begg's funnel plots for $\mathrm{rs} 2275913 \mathrm{G}>\mathrm{A}$ and $\mathrm{rs} 763780 \mathrm{~T}>\mathrm{C}$ were not symmetrical, which suggests publication bias in this meta-analysis (Figures 3 and 4).

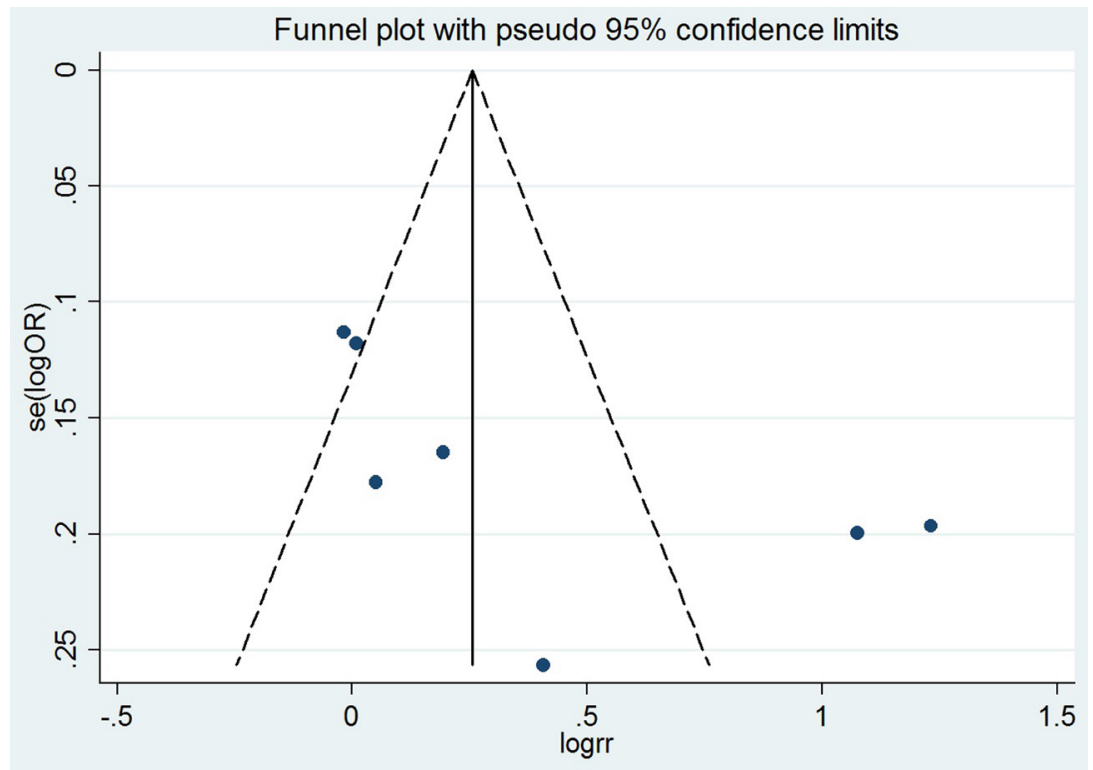

Figure 3. Funnel plots of the association between $\mathrm{rs} 2275913 \mathrm{G}>$ A polymorphism and gastric cancer risk. 


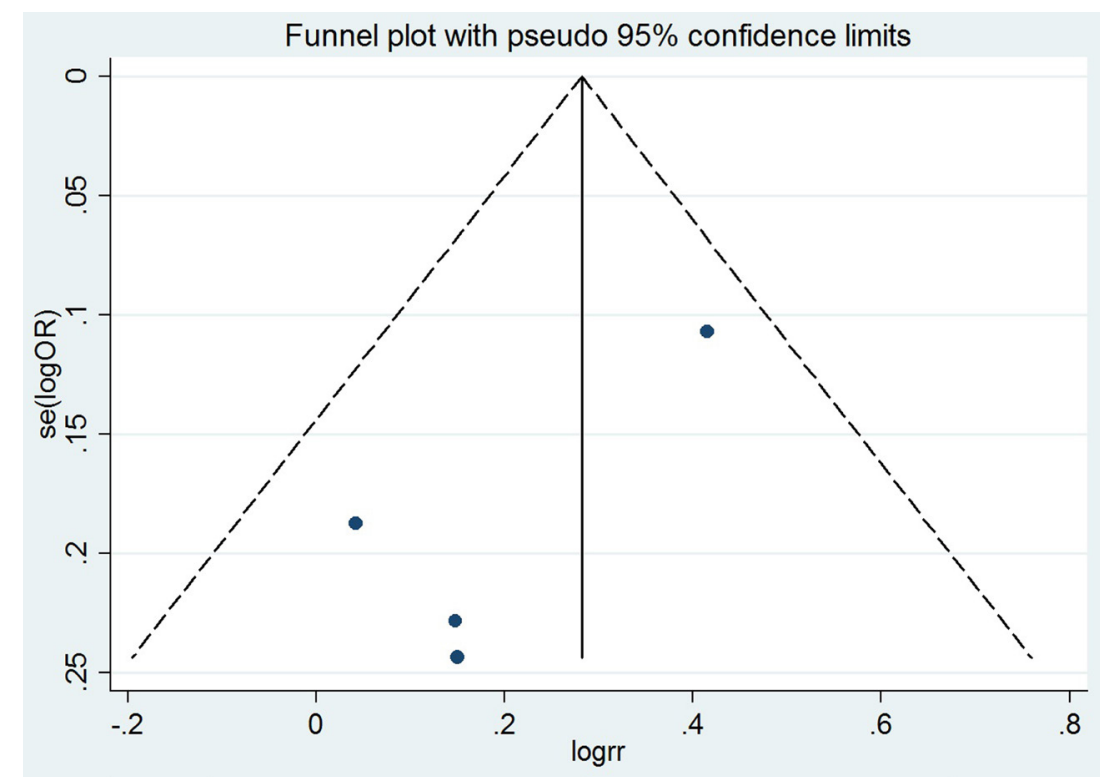

Figure 4. Funnel plots of the association between rs $763780 \mathrm{~T}>\mathrm{C}$ polymorphism and gastric cancer risk.

\section{DISCUSSION}

Previous reports have indicated that chronic inflammation may be involved in the development of gastric cancer, since IL-17A and IL-17F are expressed by Th17 cells that mediate chronic inflammation and various diseases (Hu et al., 2013; Lee et al., 2013; Park et al., 2005). Previous studies have investigated the association between $\mathrm{rs} 2275913 \mathrm{G}>\mathrm{A}$ and $\mathrm{rs} 763780 \mathrm{~T}>\mathrm{C}$ polymorphisms and the risk of gastric cancer, but the results have been inconsistent; therefore, we carried out a meta-analysis to investigate the association to obtain a reliable conclusion.

IL-17 is a relatively novel cytokine family that contains six homologous members (from IL-17A to IL-17F), which are the important inflammatory cytokines connecting innate and adaptive immunity (Kolls and Lindén, 2004). Molecular research has suggested that IL17 is an essential proinflammatory cytokine that evokes the secretion of many cytokines and chemokines by different cell types, such as mesenchymal cells and myeloid cells, to recruit monocytes and neutrophils into the microenvironment of inflammation (Iwakura et al., 2011). Furthermore, IL-17 can promote the expression of antimicrobial peptides and facilitates host defense against infections (Kao et al., 2004; Matsuzaki and Umemura, 2007).

To date, several molecular epidemiological studies have been conducted to investigate the role of rs $2275913 \mathrm{G}>\mathrm{A}$ and $\mathrm{rs} 763780 \mathrm{~T}>\mathrm{C}$ polymorphisms in the development of gastric cancer. Initially, Shibata et al. (2009) reported that the rs2275913 AA genotype was associated with a risk of gastric cancer. Chen et al. (2010) then reported that the rs $2275913 \mathrm{G}>\mathrm{A}$ polymorphism did not influence the risk of gastric cancer in a Chinese population. Subsequently, Arisawa et al. (2012), Rafiei et al. (2013), Qinghai et al. (2014), and Zhang et al. (2014) reported an association between $\mathrm{rs} 2275913 \mathrm{G}>\mathrm{A}$ and $\mathrm{rs} 763780 \mathrm{~T}>\mathrm{C}$ polymorphisms and gastric cancer risk. Our meta-analysis showed that $\mathrm{rs} 2275913 \mathrm{G}>\mathrm{A}$ and $\mathrm{rs} 763780 \mathrm{~T}>\mathrm{C}$ polymorphisms can influence the susceptibility of gastric cancer, especially in Japanese subjects. 
In our meta-analysis, heterogeneity existed but could be removed with stratified analysis; meta-regression also indicated that the ethnicity and control design might contribute to heterogeneity.

Some limitations should be considered in our study. First, although we observed a significant association between the IL-17A polymorphism and the risk of gastric cancer, the small number of published papers included in this study precluded a thorough evaluation of publication bias. Second, environmental factors are also important in the development of tumor disease. The potential interactions between genetic and environmental factors may also modify the development of gastric cancer. These factors may influence the effects of IL-17 polymorphisms on susceptibility to gastric cancer, and they should be considered. Third, different genotyping methods may also have a possible effect on the frequency of the allele. Fourth, we have only reviewed published studies, but some unpublished studies, especially negative results, may affect the final conclusion.

In conclusion, our meta-analysis is the first to indicate that the rs $2275913 \mathrm{G}>\mathrm{A}$ and rs 763780T $>$ C polymorphisms are risk factors for gastric cancer development. Moreover, future case-control and population-based studies are needed to investigate more precisely the relationships between polymorphisms and potential gene-gene and gene-environment interactions.

\section{Conflicts of interest}

The authors declare no conflict of interest.

\section{ACKNOWLEDGMENTS}

We acknowledge the help from Lung Cancer Center, Cancer Center, State Key Laboratory of Biotherapy, West China Hospital of Sichuan University.

\section{REFERENCES}

Arisawa T, Tahara T, Shiroeda H, Matsue Y, et al. (2012). Genetic polymorphisms of IL17A and pri-microRNA-938, targeting IL17A 3'-UTR, influence susceptibility to gastric cancer. Hum. Immunol. 73: 747-752.

Candido J and Hagemann T (2013). Cancer-related inflammation. J. Clin. Immunol. 33: S79-S84.

Chen J (2010). Association study of polymorphisms in i123r and il17a genes with the susceptibility of gastric cancer. Master's thesis, Nanjing Medical University, Nanjing.

Cully M (2014). Cancer: Targeting IL-17 in pancreatic cancer. Nat. Rev. Drug Discov. 13: 493.

Dai W, Zhou Q, Tan X and Sun C (2014). IL-17A (-197G/A) and IL-17F (7488T/C) gene polymorphisms and cancer risk in Asian population: a meta-analysis. Onco. Targets Ther. 7: 703-711.

$\mathrm{Hu}$ D, Hu G, Zhu J, Shen Y, et al. (2013). Association between polymorphisms of the IL-23R gene and allergic rhinitis in a Chinese Han population. PLoS One 8: e63858.

International Agency for Research on Cancer (IARC) (1994). IARC working group on the evaluation of carcinogenic risks to humans, schistosomes, liver flukes, Helicobacter pylori. IARC monographs on the evaluation of carcinogenic risks to humans, vol 61. International Agency for Research on Cancer, Lyon, 1-241.

International Agency for Research on Cancer (IARC). World Health Organization. Stomach Cancer (2014). GLOBOCAN 2012: Estimated Incidence, Mortality, and Prevalence Worldwide in 2012. Available at [http://globocan.iarc.fr/ Pages/fact_sheets_cancer.aspx]. Accessed December 2014.

Iwakura Y, Ishigame $\bar{H}$, Saijo S and Nakae S (2011). Functional specialization of interleukin-17 family members. Immunity 34: 149-162.

Kaabachi W, ben Amor A, Kaabachi S, Rafrafi A, et al. (2014). Interleukin-17A and -17F genes polymorphisms in lung cancer. Cytokine 66: 23-29.

Kao CY, Chen Y, Thai P, Wachi S, et al. (2004). Il-17 markedly up-regulates beta-defensin-2 expression in human airway 
epithelium via JAK and NF-kappaB signaling pathways. J. Immunol. 173: 3482-3491.

Kolls JK and Lindén A (2004). Interleukin-17 family members and inflammation. Immunity 21: 467-476.

Lee DY, Hong SW, Chang YG, Lee WY, et al. (2013). Clinical significance of preoperative inflammatory parameters in gastric cancer patients. J. Gastric. Cancer 13: 111-116.

Lu B and Li M (2014). Helicobacter pylori eradication for preventing gastric cancer. World J. Gastroenterol. 20: 56605665.

Matsuzaki G and Umemura M (2007). Interleukin-17 as an effector molecule of innate and acquired immunity against infections. Microbiol. Immunol. 51: 1139-1147.

Park H, Li Z, Yang XO, Chang SH, et al. (2005). A distinct lineage of CD4 T cells regulates tissue inflammation by producing interleukin 17. Nat. Immunol. 6: 1133-1141.

Qinghai Z, Yanying W, Yunfang C, Xukui Z, et al. (2014). Effect of interleukin-17a and interleukin-17f gene polymorphisms on the risk of gastric cancer in a Chinese population. Gene 537: 328-332.

Rafiei A, Hosseini V, Janbabai G, Ghorbani A, et al. (2013). Polymorphism in the interleukin-17A promoter contributes to gastric cancer. World J. Gastroenterol. 19: 5693-5699.

Scheller J, Ohnesorge N and Rose-John S (2006). Interleukin-6 trans-signalling in chronic inflammation and cancer. Scand. J. Immunol. 63: 321-329.

Shibata T, Tahara T, Hirata I and Arisawa T (2009). Genetic polymorphism of interleukin-17A and -17F genes in gastric carcinogenesis. Hum. Immunol. 70: 547-551.

van den Brandt PA and Goldbohm RA (2006). Nutrition in the prevention of gastrointestinal cancer. Best Pract. Res. Clin. Gastroenterol. 20: 589-603.

Wu X, Zeng Z, Chen B, Yu J, et al. (2014). Association between polymorphisms in interleukin-17A and interleukin-17F genes and risks of gastric cancer. Int. J. Cancer 127: 86-92.

Zarogoulidis P, Katsikogianni F, Tsiouda T, Sakkas A, et al. (2014). Interleukin-8 and interleukin-17 for cancer. Cancer Invest. 32: 197-205.

Zhang X, Zheng L, Sun Y and Zhang X (2014). Analysis of the association of interleukin-17 gene polymorphisms with gastric cancer risk and interaction with Helicobacter pylori infection in a Chinese population. Tumour Biol. 35: $1575-1580$. 